\title{
Papers to appear in forthcoming issues
}

Aaronson, J.

Abért, M. and Elek, G.

Abért, M. and Weiss, B.

Alves, J. F. and Vilarinho, H.

Ara, P., Exel, R. and Katsura, T.

Arbieto, A., Markarian, R., Pacifico, M. J. and Soares, R.

Arnaud, M.-C.

Babichev, A., Burton, R. M. and Fieldsteel, A.

Baraviera, A. T., Lopes, A. O. and Mengue, J. K.

Bergweiler, W.

Blokh, A., Curry, C. and Oversteegen, L.

Bonatti, Ch., Crovisier, S., Díaz, L. J. and Gourmelon, N.

Bowen, L. and Nevo, A.

Brémont, J. and Buczolich, Z.

Cao, Y., Hu, H. and Zhao, Y.

Chazottes, J.-R. and Collet, P.

Chazottes, J.-R., Gambaudo, J.-M., Hochman, M. and Ugalde, E.

Cheung, Y., Goetz, A. and Quas, A.

Chung, N.-P.

Conley, C. T., Kechris, A. S. and Tucker-Drob, R. D.

Cornelissen, G. and Kool, J.

Cortez, M. I. and

Rivera-Letelier, J.
Rational weak mixing in infinite measure spaces

Dynamical properties of profinite actions

Bernoulli actions are weakly contained in any free action

Strong stochastic stability for non-uniformly expanding maps

Dynamical systems of type $(m, n)$ and their $C^{*}$-algebras

Scaling rate for semi-dispersing billiards with non-compact cusps

Lower and upper bounds for the Lyapunov exponents of twisting dynamics: a relationship between the exponents and the angle of the Oseledet's splitting

Speedups of ergodic group extensions

On the selection of subaction and measure for a subclass of potentials defined by $\mathrm{P}$. Walters

Fatou-Julia theory for non-uniformly quasiregular maps

Cubic critical portraits and polynomials with wandering gaps

Internal perturbations of homoclinic classes: non-domination, cycles, and self-replication

Pointwise ergodic theorems beyond amenable groups

Maximizing points and coboundaries for an irrational rotation on a circle

Non-additive measure-theoretic pressure and applications to dimensions of an ergodic measure

Poisson approximation for the number of visits to balls in non-uniformly hyperbolic dynamical systems

On the finite-dimensional marginals of shift-invariant measures

Piecewise isometries, uniform distribution and $3 \log 2-\pi^{2} / 8$

Topological pressure and the variational principle for actions of sofic groups

Ultraproducts of measure preserving actions and graph combinatorics

Measure-theoretic rigidity for Mumford curves

Topological orbit equivalence classes and numeration scales of logistic maps 
Coutinho, R. and Fernandez, B. Extensive bounds on the topological entropy of repellers in piecewise expanding coupled map lattices

Danilenko, A. I.

A survey on spectral multiplicities of ergodic actions

Deaconu, V., Kumjian, A. and Quigg, J.

de Lima, A. and Smania, D.

Group actions on topological graphs

On infinitely cohomologous to zero observables

Del Magno, G. and Markarian, R.

Deroin, B

Dias, K.

Dirbák, M., Snoha, L'. and Špitalský, V.

A local ergodic theorem for non-uniformly hyperbolic symplectic maps with singularities

The Poisson boundary of a locally discrete group of diffeomorphisms of the circle

Enumerating combinatorial classes of the complex polynomial vector fields in $\mathbb{C}$

Minimality, transitivity, mixing and topological entropy on spaces with a free interval

Dong, P., Donoso, S., Maass, A., Shao, S. and Ye, X.

Fan, Q. and Fang, X.

Fiebig, D.

Fried, D., Marotta, S. M. and Stankewitz, R.

Gadre, V. S.

Gidea, M. and Robinson, C.

Gomes, J. B. and Ruggiero, R. O.

Gorodnik, A. and Nevo, A.

Hartman, Y.

Haydn, N., Nicol, M., Persson, T. and Vaienti, S.

Homburg, A. J.

Iommi, G. and Todd, M.

Jaerisch, J., Kesseböhmer, M. and Stratmann, B. O.

Jäger, $\mathrm{T}$.

Kenyon, R., Peres, Y. and Solomyak, B.

Kerr, D. and Nowak, P. W.

Kida, Y.

Kiriki, S. and Soma, T.

Infinite-step nilsystems, independence and complexity

Certain properties for crossed products by automorphisms with a certain non-simple tracial Rokhlin property

Canonical compactifications for Markov shifts

Complex dynamics of Möbius semigroups

Dynamics of non-classical interval exchanges

Diffusion along transition chains of invariant tori and Aubry-Mather sets

On Finsler surfaces without conjugate points

On Arnol'd's and Kazhdan's equidistribution problems

Large semigroups of cellular automata

A note on Borel-Cantelli lemmas for non-uniformly hyperbolic dynamical systems

Circle diffeomorphisms forced by expanding circle maps

Transience in dynamical systems

A Fréchet law and an Erdős-Philipp law for maximal cuspidal windings

Strange non-chaotic attractors in quasi-periodically forced circle maps: Diophantine forcing

Hausdorff dimension for fractals invariant under the multiplicative integers

Residually finite actions and crossed products

Examples of amalgamated free products and coupling rigidity

Existence of generic cubic homoclinic tangencies for Hénon maps

Kloeckner, B.

Optimal transport and dynamics of expanding circle maps acting on measures

Kosloff, Z.

The zero-type property and mixing of Bernoulli shifts 
Kułaga, J.

Kwietniak, D. and Oprocha, P.

Li, Z. and Góra, P.

Li, Z., Góra, P., Boyarsky, A., Proppe, H. and Eslami, P.

Liang, C., Sun, W. and Tian, X.

Liao, L. and Seuret, S.

Liao, L. and Steiner, W.

Lind, D., Schmidt, K. and Verbitskiy, E.

Liverani, C.

Lizana, C. and Pujals, E.

Lyons, R.

Marcus, B. and Pavlov, R.

Matui, $\mathrm{H}$.

Mayer, V. and Rempe, L.

Meyerovitch, T.

Miernowski, T. and Nogueira, A.

Möller, M. and Pohl, A. D.

Moors, W. B. and Namioka, I.

Müller, S. and Spaeth, P.

Naghmouchi, I.

Nakane, S.

Navas, A.

Osborne, J. W.

Peng, W., Yin, Y. and Zhai, Y.

Peter, J.
On the self-similarity problem for smooth flows on orientable surfaces

On weak mixing, minimality and weak disjointness of all iterates

Instability of isolated spectrum for $W$-shaped maps

Family of piecewise expanding maps having singular measure as a limit of ACIMs

Ergodic properties of invariant measures for $C^{1+\alpha}$ non-uniformly hyperbolic systems

Diophantine approximation by orbits of expanding Markov maps

Dynamical properties of the negative beta transformation

Homoclinic points, atoral polynomials, and periodic points of algebraic $\mathbb{Z}^{d}$-actions

Multidimensional expanding maps with singularities: a pedestrian approach

Robust transitivity for endomorphisms

Fixed price of groups and percolation

Approximating entropy for a class of $\mathbb{Z}^{2}$ Markov random fields and pressure for a class of functions on $\mathbb{Z}^{2}$ shifts of finite type

Some remarks on topological full groups of Cantor minimal systems II

Rigidity and absence of line fields for meromorphic and Ahlfors islands maps

Gibbs and equilibrium measures for some families of subshifts

Exactness of the Euclidean algorithm and of the Rauzy induction on the space of interval exchange transformations

Period functions for Hecke triangle groups, and the Selberg zeta function as a Fredholm determinant

Furstenberg's structure theorem via CHART groups

Helicity of vector fields preserving a regular contact form and topologically conjugate smooth dynamical systems

Pointwise-recurrent dendrite maps

Postcritical sets and saddle basic sets for Axiom A polynomial skew products on $\mathbb{C}^{2}$

An $L^{1}$ ergodic theorem with values in a non-positively curved space via a canonical barycenter map

Spiders' webs and locally connected Julia sets of transcendental entire functions

Density of hyperbolicity for rational maps with Cantor Julia sets

Hausdorff measure of escaping and Julia sets for bounded-type functions of finite order 
Petersen, C. L. and Roesch, P.

Pinto-de-Carvalho, S. and Ramírez-Ros, R.

Poirier, A.

Ponce, $\mathrm{M}$.

Quas, A. and Siefken, J.

Rousseau, J.

Schapira, B.

Sharland, T.

Sørensen, A. P. W.

Tsujii, M.

Wang, Z.

Wang, Q. and Young, L.-S.

Xavier, J.

Ye, Y.-L.

Yildiz, I. B.

Zhang, Y. and Lin, C.
Carrots for dessert

Non-persistence of resonant caustics in perturbed elliptic billiards

Hubbard forests

Towards a semi-local study of parabolic invariant curves for fibered holomorphic maps

Ergodic optimization of super-continuous functions in the shift

Recurrence rates for observations of flows

Density of half-horocycles on geometrically infinite hyperbolic surfaces

Thurston equivalence for rational maps with clusters

Geometric classification of simple graph algebras

Contact Anosov flows and the Fourier-Bros-Iaglonitzer transform

Rigidity of commutative non-hyperbolic actions by toral automorphisms

Dynamical profile of a class of rank-one attractors

Handel's fixed point theorem revisited

Ruelle operator with weakly contractive IFS

Discontinuity of topological entropy for Lozi maps

Invariant measures with bounded variation densities for piecewise area preserving maps 


\section{INSTRUCTIONS FOR CONTRIBUTORS}

\section{Editorial Policy}

The journal welcomes high quality contributions on topics closely related to dynamical systems and ergodic theory. Submissions in the field of differential geometry, number theory, operator algebra, differential, topological, symbolic, measurable dynamics and celestial and statistical mechanics are especially welcome. Expository survey papers and reviews of relevant books will be published from time to time.

\section{Submission of manuscripts}

Manuscripts should be submitted via the website: http://mc.manuscriptcentral.com/etds.

Submission of a paper is taken to imply that it has not been previously published and that it is not being considered for publication elsewhere. Authors of articles published in the journal assign copyright to Cambridge University Press (with certain rights reserved) and you will receive a copyright assignment form for signature on acceptance of your paper.

The journal strongly recommends submission of accepted papers in $\mathrm{LAT}_{\mathrm{E} X} \mathrm{X}$ using the ETDS LATEX class file. Papers that use this class file will be processed more efficiently. A LATEX2e file etds.cls is available via anonymous ftp from the Cambridge University Press site at ftp.cup.cam.ac.uk in the directory /pub/texarchive/journals/latex/etds-cls/. In case of difficulties with these files, please contact etds@ sunrise-setting.co.uk or the Journal editorial office at etds@maths.warwick.ac.uk. Alternatively, authors may use 'article' style.

On acceptance of a paper, authors should upload the $\mathrm{LAT}_{\mathrm{E}} \mathrm{X}$ source code including the figures (line figures only) and all author-defined macro and style files, together with a pdf produced using the same file, via the submission site http://mc.manuscriptcentral.com/etds.

The publisher reserves the right to typeset any article by conventional means if the author's $\mathrm{T}_{\mathrm{E}} \mathrm{X}$ code presents problems in production.

\section{Manuscript}

Papers should be typed with generous margins. The pages must be numbered.

The first page should give the title, the author's name and institution, and a short abstract intelligible to mathematicians.

The title, while brief, must be informative (e.g. 'A new proof of the ergodic theorem', whereas 'Some applications of a theorem of Birkhoff' would be useless).

\section{Notation}

Avoid abbreviations such as Thm, Prop., Eq., iff. In the text do not use symbols $\forall, \exists, \Rightarrow$ and $\Leftrightarrow$. Fractions are generally best expressed by a solidus. Complicated exponents like $\exp \left\{z^{2} \sin \theta /\left(1+y^{2}\right)\right\}$ should be shown in this and no other way.

It helps if displayed equations or statements which will be quoted later are numbered in order on the right of their line. They can then be referred to by, for example, 'from (7)'.

If an author wishes to mark the end of the proof of a theorem, the sign $\square$ may be used.

Footnotes should be avoided.

\section{Figures}

Graphics should be prepared to professional standards, preferably using Postscript or LATEX drawing facilities. Each text figure must be numbered as Figure 1, Figure 2, $\ldots$ and its intended position clearly indicated in the manuscript. Figures should be used sparingly and only when they greatly clarify the exposition. The preferred resolutions for submission of electronic artwork are: halftone images $300 \mathrm{dpi}$; line tone $600 \mathrm{dpi}$; bitmap $1200 \mathrm{dpi}$.

Tables

Tables should be numbered (above the table) as Table 1 , Table $2, \ldots$ Indicate the position of each in the text as for figures.

\section{References}

References should be collected at the end of the paper numbered in alphabetical order of the author's names or by order of citation. Include in the list of references only those works that are cited. For the style of references please consult recent issues of the journal. A reference to a book should give the title, in italics, and then in roman type the publisher's name and the place and year of publication: [4] N. Dunford and J. T. Schwartz. Linear Operators. Part I. Wiley, New York, 1958.

A reference to a paper should give in italics the title of the periodical, the number of the volume and year, and the beginning and end pages of the paper. Journal titles should be abbreviated as in Mathematical Reviews:

[6] J. E. Littlewood. The 'pits effect' for functions in the unit circle. J. Analyse Math. 23 (1970), 236-268.

\section{Proofs}

Authors receive one pdf proof for correction. Typographical and minor corrections only are permitted at this stage. For papers with more than one author the proofs are sent to the first named author unless the editor receives other instructions. It is important that proofs are corrected and returned promptly.

\section{Offprints}

No paper offprints are provided, but the corresponding author will be sent the pdf of the published article. Print offprints may be purchased at extra cost at proof stage.

This journal issue has been printed on FSC-certified paper and cover board. FSC is an independent, non-governmental, not-for-profit organization established to promote the responsible management of the world's forests. Please see www.fsc.org for information. 


\title{
Ergodic theory and dynamical systems
}

\author{
VOLUME 32 PART 4 AUGUST 2012
}

\section{CONTENTS}

Barański, K., Karpińska, B. and Zdunik, A. Bowen's formula for meromorphic functions

Bárány, B. Dimension of the generalized 4-corner set and its projections

Biswas, K. Simultaneous linearization of germs of commuting holomorphic diffeomorphisms

Carlsen, T. M. and Thomsen, K. The structure of the $C^{*}$-algebra of a locally injective surjection

Carminati, C. and Tiozzo, G. A canonical thickening of $\mathbb{Q}$ and the entropy of $\alpha$-continued fraction transformations

Demers, M. F., Wright, P. and Young, L.-S. Entropy, Lyapunov exponents and escape rates in open systems

Farina, A. and Valdinoci, E. Some results on minimizers and stable solutions of a variational problem

Guivarc'h, Y. and Raja, C. R. E. Recurrence and ergodicity of random walks on linear groups and on homogeneous spaces

Jiang, M. Differentiating potential functions of SRB measures on hyperbolic attractors

Killough, D. B. and Putnam, I. F. Ring and module structures on dimension groups associated with a shift of finite type

Li, Y., Chen, E. and Cheng, W.-C. Tail pressure and the tail entropy function

Mihaljević-Brandt, $H$. Dynamical approximation and kernels of nonescaping hyperbolic components

Moss, A. and Walkden, C. P. Stable topological transitivity properties of $\mathbb{R}^{n}$-extensions of hyperbolic transformations

Reeve, H. W. J. The packing spectrum for Birkhoff averages on a self-affine repeller

Schneider, M. Alexandrov-embedded closed magnetic geodesics on $S^{2}$

Papers to appear in forthcoming issues 